\title{
Trigeminal Neuralgia - A Case Report with Review of Literature
}

\author{
Saraswathi G K ${ }^{1}$ and Swathi $\mathrm{T}^{2^{*}}$ \\ 1Proffesor and Head of the Department of Oral Medicine and Radiology, Meenakshi Ammal Dental College, Chennai, Tamil Nadu, \\ India \\ 2Department of Oral Medicine and Radiology, Meenakshi Ammal Dental College, Chennai, Tamil Nadu, India
}

*Corresponding author: Dr. Swathi T, PG, Department of Oral Medicine and Radiology, Meenakshi Ammal Dental College, Chennai, Tamil Nadu, India. Tel: 9962484011, E-mail: Swathi.march91@gmail.com

Citation: Swathi T (2017) Trigeminal Neuralgia - A Case Report with Review of Literature. SAJ Case Report 4: 102

Article history: Received: 23 December 2016, Accepted: 16 January 2017, Published: 20 January 2017

\begin{abstract}
Trigeminal neuralgia also known as 'Fothergill's disease' or 'tic douloureux' is a very peculiar disease. The severe pain is paroxystic and can be triggered by a mild cutaneous stimulus on the face or "trigger zone". It can be classified based on its etiology and symptoms. The disease has a wide variety of etiology and clinical presentation. Differential diagnosis of idiopathic facial pain, migraine, temporal arteritis, glossopharyngeal neuralgia can be considered. The management will include both medical and a surgical approach. We present a case of trigeminal neuralgia of the maxillary and mandibular division along with clinical history, MRI (Magnetic Resonance Imaging) findings and treatment approach.
\end{abstract}

Keywords: Trigeminal Neuralgia; MRI; Resonance Theory; Peripheral Neurectomy

\section{Introduction}

The largest cranial nerve is the trigeminal nerve which got its name - "trigeminal" - is derived from the fact that each nerve, one on each side of the pons, has three major branches: the ophthalmic nerve (V1), the maxillary nerve (V2) and the mandibular nerve (V3). Purely sensory are the ophthalmic and maxillary nerves, the mandibular nerve has both sensory and motor functions. Trigeminal neuralgia also known as 'Fothergill's disease' (named after john fothergill) or 'tic douloureux' is a very peculiar disease [1]. The severe pain is paroxystic and can be triggered by a mild cutaneous stimulus on the face or "trigger zone". There is no specific etiology that is identified till date and can be attributed to a number of reasons.

\section{Case Report}

A patient age 56/F reported to our department with the chief complaint of severe pricking type of pain which is intermittent on the right side of the face for the past two years. She revealed history of pain that aggravates while eating, swallowing, washing her face, clenching her teeth and even on touching. She doesn't experience pain while sleeping. Patient described the pain as severe, unbearable, intermittent, shock like and unilateral. Past dental history revealed trigeminal neuralgia which was treated with carbamazepine $100 \mathrm{mg}$ twice daily, patient was relieved of symptoms for a period of one year. She however continued medication irregularly and started to experience the similar pain for the past six months (Figure 1 and 2).

Her medical history revealed that she had hypertension for the past 10 years and is under medication her blood pressure was $134 / 84 \mathrm{mmHg}$. On examination the patient was moderately nourished and responded to time and place, patient is married and has two kids. On extra oral examination pain was present on touching the supraorbital, infraorbital, zygomatic, nasolabial fold, ala and tip of nose, upper and lower lip, pre auricular and angle of mandible on the right side. On intraoral examination pain was present on palpation of maxillary and mandibular alveolar ridge, right side of the tongue and hard palate. Her permanent molars on the right side were extracted. Patient jerked from the dental chair while eliciting pain.

Patients OPG revealed no of teeth: 26, with generalized loss of normal crown contour suggestive of generalized attrition. Styloid process length was evaluated to rule out eagle's syndrome. A diagnostic block was given first for the mandibular division of the trigeminal nerve followed by the maxillary division and patient was immediately free of pain. Thus a diagnosis of trigeminal neuralgia involving the maxillary and mandibular division on the right side was established (Figure 3). 


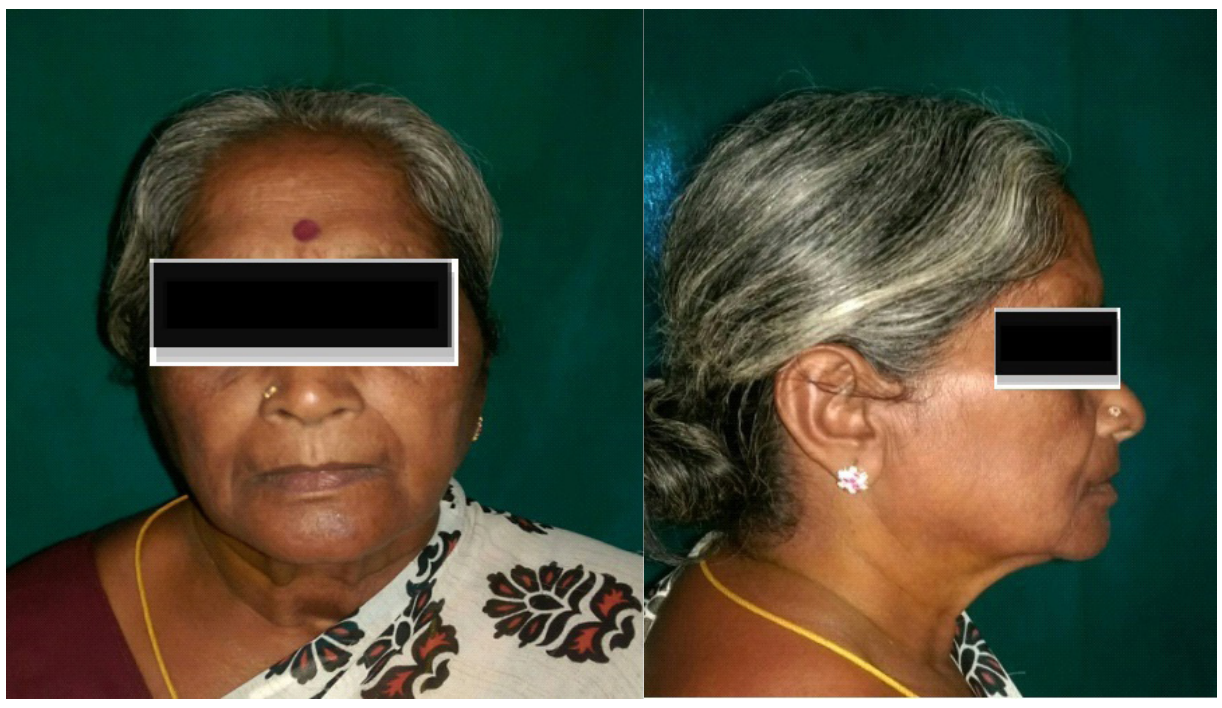

Figure 1: Extra Oral

Figure 2: Extra Oral

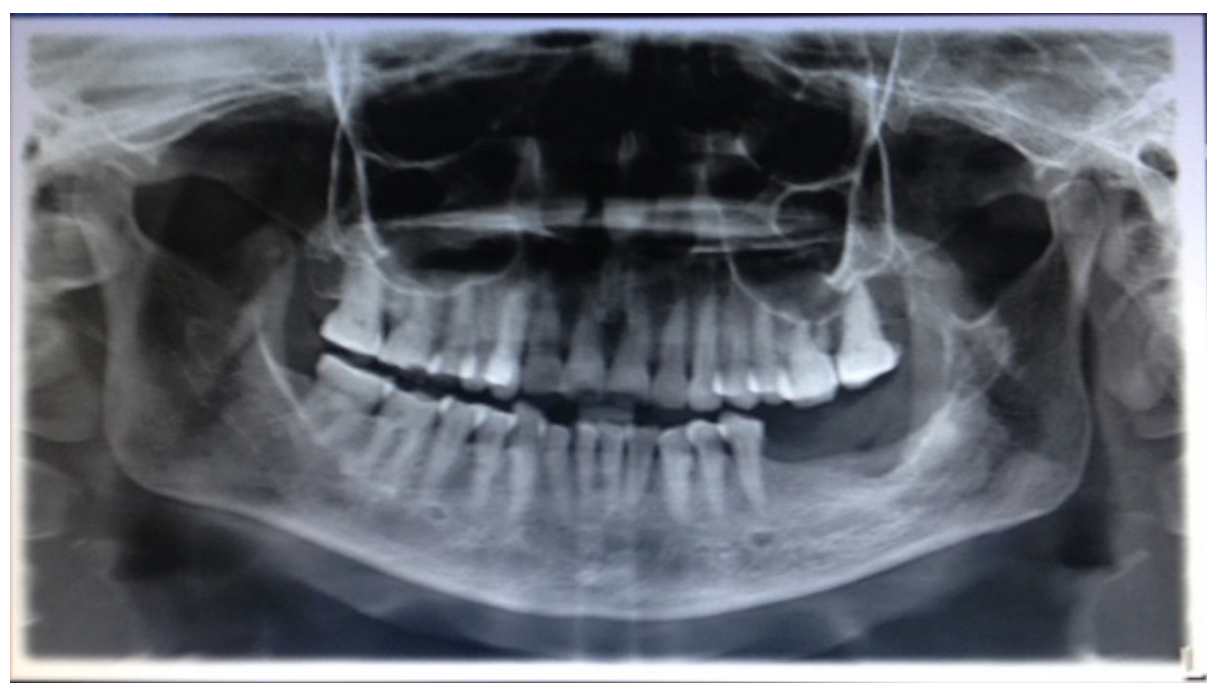

Figure 3: OPG

MRI revealed normal brain parenchyma with a small branch of vertebral artery seen intending and looping over the root exit zone of cisternal segment of right trigeminal nerve (Figure 4 and 5). The patient was treated with peripheral neurectomy of the maxillary and mandibular division of trigeminal nerve and is currently free from symptoms and is under regular follow up.

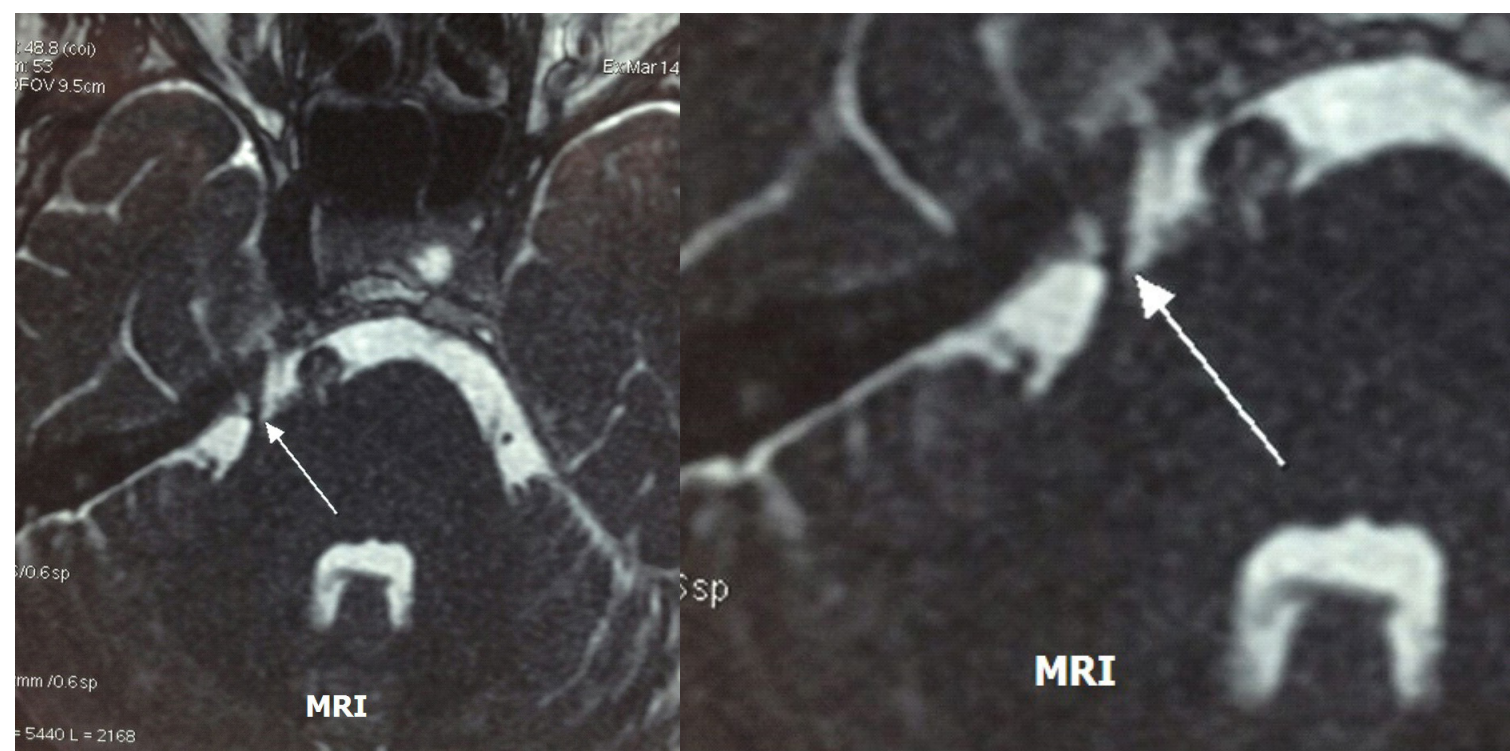

Figure 4, 5: A small branch of vertebral artery seen intending and looping over the root exit zone of cisternal segment of right trigeminal nerve 


\section{Discussion}

Kugelberg and Lindblom in 1959 stated trigeminal neuralgia is characterized by brief paroxysms of pain, limited to the facial distribution of the trigeminal nerve and precipitated by stimuli to sensory endings in the trigeminal receptive area [2]. International Association for the Study of Pain (IASP) defined trigeminal neuralgia as "sudden usually unilateral severe brief stabbing recurrent pain in the distribution of one or more branches of the 5th cranial nerve". Similarly International Headache Society (IHS) defined it as "painful unilateral affliction of the face characterized by brief electric shock like pain limited to the distribution of one or more divisions of trigeminal nerve" [3,4].

Trigeminal neuralgia has been classified Based on etiology into two types by the international headache society (IHS) [5].

1. Classical - is caused by vascular compression of the nerve; the superior cerebellar artery is most often responsible for neurovascular compression on the trigeminal nerve, although other arteries or veins may also be responsible.

2. Symptomatic - it has the same clinical criteria, but another underlying cause except vascular compression is responsible for the symptoms. And based on the symptoms it is classified as

a. Typical Trigeminal Neuralgia (Tic Douloureux): which is the most common type of trigeminal neuralgia. It presents with minor aching or burning pain within the affected distribution of the trigeminal nerve.

b. Atypical trigeminal neuralgia is characterized by a unilateral, continuous and severe aching or burning pain. Both these types of pain can occur in the same patient, it can share the same time also [6,7].

The etiological factors may be grouped into three major theories [8]. 1) Disease related 2) Direct injury to the nerve and 3) Poly etiologic origin although compression of the trigeminal nerve root is considered the most common cause of neuralgia nerve (Table 1).

\begin{tabular}{|c|c|c|c|}
\hline \multirow{2}{*}{$\begin{array}{l}\text { Related to } \\
\text { systemic } \\
\text { Diseases }\end{array}$} & \multicolumn{2}{|c|}{ Injury to Trigeminal nerve Directly } & \multirow{2}{*}{$\begin{array}{l}\text { Idiopathic } \\
\text { Causes }\end{array}$} \\
\hline & Peripheral Nerve & Central Nerve & \\
\hline Multiple & $\begin{array}{l}\text { "Allergic Hypothesis" } \\
\text { infection, ENT } \\
\text { pathology etc., }\end{array}$ & \multirow{2}{*}{$\begin{array}{l}\text { "Neurovascular compression } \\
\text { Hypothesis" arteriovenous } \\
\text { malformation, malformation, } \\
\text { meningiomas. Schwannomid } \\
\text { cyst, aneurysms, } \\
\text { terbeculomas etc., }\end{array}$} & \multirow{2}{*}{$\begin{array}{l}\text { Conditions } \\
\text { that evoke } \\
\text { dystropy and } \\
\text { demyelination }\end{array}$} \\
\hline $\begin{array}{l}\text { diseases, } \\
\text { rheumatism } \\
\text { etc., }\end{array}$ & $\begin{array}{l}\text { "Compression } \\
\text { syndrome hypothesis" } \\
\text { due to narrowing of the } \\
\text { canal, trauma etc }\end{array}$ & & \\
\hline
\end{tabular}

Table 1: Etiological factors causing trigeminal neuralgia

Another etiological factor identified in the recent past include diabetes which causes damage to tiny peripheral arteries in turn leads to decreased circulation of blood and there by nerve fiber impairment. It may be a reason for neuralgia anywhere in the body. Infectious conditions caused by Mycobacterium leprae, Secondary syphilis, Leptospirosis, Shigella etc., can also lead to this type of neuralgia. Allergic reaction and its frequent manifestations such as cold, tonsillitis, maxillary sinusitis, chronic rhinitis, and chronic inflammation can trigger immune response increasing the amount of histamine and IgE secretion. This immune response results in accumulation immunoglobulins and histamine in the trigeminal nerve region which plays a role in the pathogenesis of trigeminal neuralgia. Recently the resonance theory as gained popularity in the etiology of trigeminal neuralgia; according to the resonance theory the frequency and the vibrations of the structures that surrounds the trigeminal nerve becomes close to its natural frequency. The resonance of the trigeminal nerve resonates along, matching the same frequency; this resonance can cause impairment of the nerve fibers and leads to abnormal transmission of the impulse which eventually results in facial neuralgia $[9,10]$.

Symonds stated that trigeminal neuralgia proceeds with prodromal symptoms and it was named as "Pre-trigeminal neuralgia" by Mitchell [3,11]. The prodromal symptoms present with dull, aching, and continuous pain in the maxilla and mandible and later develop into the classical paroxysmal pain. The prodromal symptoms present with toothache or sinusitis-like pain lasting for several hours, and most of the times it is triggered by jaw movements or hot or cold food stuffs. The pre trigeminal neuralgic pain subsides on taking carbamazepine or baclofen during the prodromal period itself. It also decreases the severity of the disease [10]. Classical trigeminal neuralgia symptoms presents with pain attacks that last for few seconds or it may occur in "volleys" - that is multiple bursts of pain in quick intervals - lasting minutes [3] (Table 2 and 3).

\begin{tabular}{|c|c|}
\hline Character: Shooting,sharp Terrifying,exhausting Unbearable & Provoking: Light touch (eating, talking) Spontaneous \\
\hline Severity : Moderate Severe & Relieving : Sometimes sleep Drugs \\
\hline Trigeminal nerve: Right $(60 \%)$ Rarely $1^{\text {st }}$ division & Associated: Trigger points Sensory change \\
\hline Site: Unilateral & Timing: Periods of remission Periods of exacerbation \\
\hline
\end{tabular}

Table 2: Classical clinical features of trigeminal neuralgia 
1. Paroxysmal attacks of facial pain which last a few seconds to less than two minutes.

2. Pain has at least 4 of the following characteristics:

- Distribution along one or more divisions of the trigeminal nerve.

- Sudden, intense, sharp, superficial, stabbing or burning in quality.

- Pain intensity is severe.

- Precipitation from trigger areas, or by certain activities such as eating, talking, washing the teeth or cleaning the face.

- Between paroxysms the patient is entirely asymptomatic.

3. Attacks are stereotyped in the individual patient.

4. No neurological deficit and exclusion of other causes.

Table 3: The diagnostic criteria of the International Headache Society (IHS) (1988) [3]

Common Differential diagnosis includes glossopharyngeal neuralgia, dental infection or cracked tooth syndrome, temporomandibular joint pain, atypical facial pain, migraine and temporal arthritis [12].

The medical management includes drugs; carbamazepine is the first drug of choice for trigeminal neuralgia. Dose of 100 to $200 \mathrm{mg}$ is started for two or three time daily. Doses should be increased and titrated to the severity of the pain up to a maximum dose of $1200 \mathrm{mg}$ /day. Serum level is a useful way of monitoring dose of carbamazepine (6 to $12.5 \mathrm{ug} / \mathrm{ml}$ ) [13,14]. If the symptoms doesn't subside other drugs should be added this include baclofen or phenytoin, sodium valproate, gabapentin, lamotrigine, and clonazepam. Lamotrigine has recently been validated as the drug of choice for trigeminal neuralgia induced by multiple sclerosis.

The surgical management of trigeminal neuralgia is resorted as a treatment only when the medical management does not provide relief for the patient and pain continuous to manifest its severity. Just as our patient experienced severe pain even after a trial of various drugs. The surgical management include the following procedures Microvascular decompression, Glycerol gangliolysis, Radiofrequency gangiolysis, Balloon compression, Stereotactic radiosurgery, peripheral neurectomy, Cyber knife in trigeminal neuralgia etc., $[7,15,16]$. The choice and prognosis of surgical management depends upon the etiology of the disease and also patient considerations like age and associated diseases.

\section{Conclusion}

Trigeminal neuralgia is still considered a life threatening disease and this can be attributed to the severity and intensity of the pain the patient experiences; Thus the right approach to treat such patients will be to focus on relieving the symptoms caused by the disease. The diagnosis can be made on the history and the clinical symptoms and is best treated with medication. When the medical management fails surgical approach can be considered.

\section{References}

1. J M S Pearce. (2005) Historical note on Trigeminal neuralgia (Fothergill's disease) in the 17th and 18th centuries, J Neurol Neurosurg Psychiatry 18. 5-6.

2. Eric Kugelberg and Ulf Lindblom (1959) The Mechanism of the Pain in Trigeminal Neuralgiaby. J Neurol Neurosurg Psychiatry 22. 36-43.

3. Rahul Srivastava - Srivastava et al., (2015) Diagnostic criteria and management of trigeminal neuralgia: A review Asian Pac. J. Health Sci 2. 108-18.

4. R W hurt. (2009) Textbook of sterotactic and functional neurosurgery, pathophysiology of trigeminal neuralgia 2359-419.

5. Krafft RM. (2008) Trigeminal neuralgia. Am Fam Physician 77.9

6. Cohen J. (2014) Trigeminal Neuralgia. Danbury (CT): The National Organization for Rare Disorders.

7. T.J.Nurmikko. (2001) Trigeminal neuralgia-pathophysiology, diagnosis and current treatment. Br j anaesthesia 87.117-32.

8. Gintautas Sabalys, Gintaras Juodzbalys, Hom-Lay Wang (2012) Aetiology and Pathogenesis of Trigeminal Neuralgia: a Comprehensive Review, J Oral Maxillofac Res 3. E2.

9. Katsuhiro Toda. (2007) Etiology of Trigeminal Neuralgia, Japanese stomatology society. 4. 10-8.

10. Joffroy A, Levivier M, Massager N. Trigeminal neuralgia. Pathophysiology and treatment. Acta Neurol Belg 1. 20-5.

11. Sanjeev Jindal and Anshu Singla (2016) Pretrigeminal neuralgia. Br Dent J. 149. 167-70.

12.(1949) Symonds C. Facial pain. Ann R Coll Surg Engl. 4. 206-12.

13. G. Cruccu (2008) AAN-EFNS guidelines on trigeminal neuralgia management. Eur J Neurol 15. 1013-28.

14. Srivatava. (2007) Mechanism of pain control, j.neurosurgery 3. 14-6.

15. R W hurt. (2011) Textbook of orofacial pain, Trigeminal Neuralgia Surgical Aspects.

16. Mitchell. (2016) Trigeminal Neuralgia Treatment \& Management, Drugs \& Diseases. 